\title{
A Case of Polyneuropathy Following a Single Dose of Diethylcarbamazine and Albendazole During Mass Administration of Drug - A Case Report
}

\author{
Kohli SC, ${ }^{1^{*}}$ Shreshta UK, ${ }^{1}$ Alurkar VM,,${ }^{1}$ Maskey $A,{ }^{1}$ Parajuli M, ${ }^{1}$ Paudel $N,{ }^{1}$ Pradhananga $S,{ }^{1}$ Vaidya $S^{1}$ \\ ${ }^{1}$ Department of Medicine, Manipal College of Medical Sciences, Pokhara, Nepal
}

\author{
* Corresponding Author: \\ Prof. Suresh Chandra Kohli \\ Department of Medicine \\ Manipal Teaching Hospital, Pokhara, Nepal \\ Email: profsckohli@rediffmail.com
}

\section{Citation}

Kohli SC, Shrestha UK, Alurkar VM, Maskey A, Parajuli $M$, Poudel N, Pradhananga S, Vaidya S. Polyneuropathy following a single dose of Diethylcarbamazine and Albendazole during mass administration of drug - a case report. Nepal Journal of Medical Sciences. 2012; 1(1): 56-58.

\begin{abstract}
The global program to eliminate Lymphatic Filariasis created by The World Health organization in 1997 is based on mass administration of single annual doses of diethylcarbamazine ( DEC) plus albendazole in non African regions and of albendazole plus ivermectin in Africa. The usual side effects of DEC treatment include fever, chills, arthralgia, headaches, nausea, and vomiting. Albendazole is associated with relatively few side effects consisting of occasional nausea, vomiting, abdominal pain, headache, reversible alopecia, elevated aminotransferases and rarely leucopenia and rash. We report a case of polyneuropathy in a young individual following DEC and albendazole during mass drug administration.
\end{abstract}

Keywords: Albendazole; DEC; Polyneuropathy.

\section{Introduction:}

Lymphatic filariasis is targeted for elimination globally through mass drug administration (MDA) with Diethylcarbamazine (DEC), or ivermectin monotherapy or either drug in combination. ${ }^{1}$ Adverse reaction to DEC treatment may represent either an acute hypersensitivity reactions to antigens released from dead or dying parasites or inflammatory reaction induced by regimen of treatment of lymphatic filariasis. ${ }^{2}$ Cases of severe encephalopathy have been reported with DEC or ivermectin and DEC plus albendazole treatment ${ }^{3-6}$ and one case of encephalopathy has also been reported with albendazole administration alone. ${ }^{7}$ No case of Polyneuropathy have been reported so far following administration of single dose of DEC and albendazole in the literature. We report a case of polyneuropathy following single dose of DEC and albendazole during mass drug administration.

Case report: A 20 years healthy young male hailing from Syangjha, presented with progressive weakness of both upper and lower limbs of six days duration following the consumption of DEC and albendazole during mass distribution of drug. At the onset, next day after taking the drugs, he started weakness of both the upper limbs followed one day later by the weakness of both the lower limbs. There was no history of fever or diarrhea preceeding the illness. There were no sensory complaints or sphincter disturbances. He did not give history of any similar illness in the past.

On examination, his general condition was good, afebrile, vitals paramaters were normal. In the examination of the Central Nervous system, Higher Mental functions and cranial nerves were normal. There was no wasting; there was hypotonia in both upper and lower limbs. The Power was $3 / 5$ in both the upper limbs and 4/5 in both the lower limbs. Deep tendon reflexes were absent in all the four limbs and plantar response was absent bilaterally. Sensory examination was normal. There were no signs of meningial irritation. Examination of other systems was normal.

Laboratory investigations revealed: $\mathrm{Hb}: 15.4$ gm\%, WBC:14200/cc, Platelets:-289000/cc and peripheral Blood smear was normal. Cerebrospinal fluid was clear, not under increased pressure. Biochemical examination showed:Glucose: $67 \mathrm{mg} \%$, Protein: $18 \mathrm{mg} \%$. Cytology showed Cell count of 3 lymphocytes, Gram stain and AFB stain were negative. Serum $\mathrm{Na}^{+} 146 \mathrm{meq} / \mathrm{L}, \mathrm{K}^{+} 4.3 \mathrm{meq} / \mathrm{L}$, Blood urea 
$45 \mathrm{mg} \%$, Serum Creatinine $0.8 \mathrm{mg} \%$. HIV I and II, Anti $\mathrm{HCV}, \mathrm{HBs} A \mathrm{~g}$ were negative.

With the above history, clinical findings and laboratory parameters, a diagnosis of acute inflammatory demyelinating polyneuropathy was made. The patient was kept under observation in the intensive care unit and managed symptomatically. The further course of the illness was uneventful. He showed gradual improvement in power in all the four limbs after 2 days of admission and recovered significantly after 5 days. At discharge his power in all the four limbs was $4+/ 5$.

Discussion: Community-based intervention is the current approach to elimination of lymphatic filariasis as a public health problem. The underlying tenet of this approach is that mass annual distribution of antimicrofilarial chemotherapy albendazole with DEC in non-African regions will profoundly suppress microfilaremia. If the suppression is sustained, then transmission can be interrupted.

Side effects of DEC treatment include fever, chills, arthralgias, headaches, nausea, and vomiting. ${ }^{1}$ Albendazole is associated with relatively few side effects consisting of nausea, vomiting, abdominal pain, headache, reversible alopecia, elevated aminotransferases and rarely leucopenia and rash. ${ }^{1}$ In patients infected with L. loa, who have high levels of Loa microfilaremia, DEC-like ivermectin can elicit severe encephalopathic complications. ${ }^{1,3-6}$ Only one case of encephalopathy has been reported following Loa loa treatment with albendazole. ${ }^{7}$ Both the development and the severity of these reactions are directly related to the number of microfilariae circulating in the bloodstream. ${ }^{1}$, 8-12 The adverse reactions may represent either an acute hypersensitivity reaction to the antigens being released by dead and dying parasites or an inflammatory reaction induced by the intracellular Wolbachia endosymbionts freed from their intracellular niche. ${ }^{1}$ This observation implies that drug toxicity is not the direct cause of the side effects, but rather that the host inflammatory response to dying microfilariae is the chief determinant. ${ }^{13-17}$

Haarsbrink $\mathrm{M}$ et al ${ }^{18}$ reported that in patients treated with DEC ,its Plasma concentrations were not different among patients suffering from varying degrees of illness. Interleukin (IL)-6, IL-10, lipopolysaccharide-binding protein (LBP), and soluble tumor necrosis factor receptors (sTNF-Rs) increased after treatment with DEC. IL-6 and LBP, however, showed the strongest association with adverse reactions. Increasing levels of these molecules were closely correlated with the mounting severity of adverse reactions, which raises the possibility that they play an important role in systemic inflammation that arises after DEC treatment of filarial patients.

In another study ${ }^{19}$ Haarsbrink et al reported that after initiation of treatment, the levels of IL-6 and LPS-binding protein (LBP) were consistently and significantly higher in microfilaraemics who suffered most from adverse reactions compared with endemic normals and elephantiasis. These results demonstrate that intake of DEC leads to an increase in a selected number of inflammatory mediators in the group of filarial patients who suffer most from adverse systemic reactions patients.

Several lines of evidence support an autoimmune basis for acute inflammatory demyelinating polyneuropathy. It is likely that both cellular and humoral immune mechanisms contribute to tissue damage in acute inflammatory demyelinating polyneuropathy. T cell activation is suggested by the finding that elevated levels of cytokines and cytokine receptors are present in serum interleukin (IL) 2,soluble IL 2 receptor and in cerebrospinal fluid IL-6,tumour necrosis factor, interferon. ${ }^{1}$

In view of above observations, it is postulated that an autoimmune basis may also be responsible for polyneuritis in this patient. In conclusion, a case of polyneuropathy following single dose of DEC and albendazole during MDA has been presented. Pretreatment status of blood for microfilarae is not available as it was not done during MDA.

\section{References:}

1. Bockcarie MJ, Deb RM.Elimination of lymphatic filariasis : do we have the drugs to complete the job ? Curr Opin Infect Dis 2010;23:617-20.

2. Nutman TB, Weller P. Filarial and Related infections. In Longo DL, Kasper DL, Jameson JL editors. Harrison's Principles of Internal Medicine. $18^{\text {th }}$ edition. Mc Graw Hill. NewYork, 2012;1:1745-8.

3. Carme B, Boulesteix J, Boute MF, et al. Five cases of encephalitis during treatment of loisis with dietylcarbamazine. Am J Trop Med 1991;44:684-90.

4. Gordon.J, Gordon-W, Ngangue N, et al. Serious reaction after mass treatment of Onchocerciasis with ivermectin in an area endemic for Loa loa infection, Lancet 1997;350:18-22.

5. Bouquinesq, Gardon M ,Gardon J, et al. Three probable cases of Loa loa encephalopathy following ivermectin treatment for onchocerciasis. Am J Trop Med Hyg 1998;58:684-90.

6. Youngyuth $\mathrm{P}$, Koyadun S, Jatuarabundit $\mathrm{N}$, et al. Adverse reaction of $300 \mathrm{mg}$ diethlcarbamazine and in 
Case Report | Kohli SC, et al. Polyneuropathy following a single dose of Diethylcarbamazine.

combination of $400 \mathrm{mg}$ albendazole for a mass annual treatment in migrant workers in Phang Aga Province. J Med Assoc 2007;90:552-63.

7. Blum J, Wiestner A, Fuhr P, et al. Encephalopathy following Loa loa treatment with albendazole. Acta Tropica 2001;78:63-5.

8. Horton J. Albendazole: a review of anthelmintic efficacy and safety in humans. Parasitology 2000;121:113-32.

9. Pani S, Subramanyam RG, Das L, et al. Tolerability and efficacy of single dose albendazole, diethylcarbamazine citrate (DEC) or co-administration of albendazole with DEC in the clearance of Wuchereria bancrofti in asymptomatic microfilaraemic volunteers in Pondicherry, South India: a hospital-based study. Filaria J 2002; 1:1.

10. El Setouhy M, Ramzy RM, Ahmed ES, et al. A randomized clinical trial Med Public Health 2005;36:832-40.

11. Haarbrink M, Terhell AJ, Abadi GK, et al. Adverse reactions following diethylcarbamazine (DEC) intake in 'endemic normals', microfilaraemics and elephantiasis patients. Trans R Soc Trop Med Hyg 1999;93:91-6.

12. Supali T, Ismid IS, Rückert $P$, et al. Treatment of Brugia timori and Wuchereria bancrofti infections in Indonesia using DEC or a combination of DEC and albendazole: adverse reactions and short-term effects on microfilariae. J Commun Dis 2004;36:240-50.
13. Piessens WF, Beldekas M. Diethylcarbamazine enhances antibody-mediated cellular adherence to Brugia malayi microfilariae . Nature 1979;282:845-7.

14. Cesbron J-Y, Capron A, Vargaftig BB, et al. Platelets mediate the action of diethylcarbamazine on microfilariae. Nature 1987;325:533-6.

15. Campbell WC, ed. Ivermectin and abamectin. New York: Springer-Verlag 1989:363.

16. Ottesen EA. Description, mechanisms and control of reactions to treatment in the human filariases . Ciba Found Symp 1987;127:265-83.

17. Eric A, Ottesen M.D, Vijayasekaran V et al. A controlled trial of Ivermectin and Diethylcarbamazine in lymphatic filariasis. N Engl J Med 1990;322:1113-7.

18. Haarbrink M, Abadi GK, Buurman WA, et al. Strong association of interleukin-6 and lipopolysaccharidebinding protein with severity of adverse after diethylcarbamazine treatment of microfilaremic patients : J Infect Dis. 2000;182:564-9.

19. Haarbrink M,Terhell AJ, Abadi GK, et al. Inflamatory cytokines following diethylcarbamazine(DEC) treatmentof of different clinical groups in lymphaticfilariasis : Trans R Soc Trop Med Hyg 1999;93:665-72. 